\title{
Barreras para la atención en salud del cáncer gástrico, Santander, Colombia. Etapa exploratoria
}

\section{Barriers to Health Care for Gastric Cancer Patients in Santander, Colombia: Exploratory Stage}

Claudia Janeth Uribe P., Angélica María Amado N., ${ }^{2}$ Ana María Rueda P., ${ }^{3}$ Leydi Yurani Mantilla V. ${ }^{3 *}$

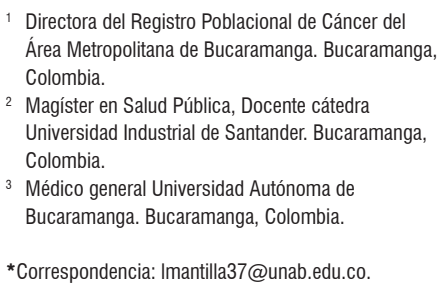

2 Magíster en Salud Pública, Docente cátedra Universidad Industrial de Santander. Bucaramanga, Colombia.

${ }^{3}$ Médico general Universidad Autónoma de

Bucaramanga. Bucaramanga, Colombia.

*Correspondencia: Imantilla37@unab.edu.co.

\begin{abstract}
Resumen
Introducción: el cáncer gástrico es un problema de salud pública; ocupa el quinto y tercer lugar en incidencia y mortalidad mundial, respectivamente. Objetivo: describir las barreras para la atención en salud percibidas por el adulto con cáncer gástrico, su cuidador y el médico tratante en el departamento de Santander, Colombia-etapa exploratoria en el período 2015-2016. Metodología: estudio cualitativo utilizando algunas técnicas del proceso de análisis de la teoría fundamentada a partir de la aplicación de entrevistas semiestructuradas que fueron codificadas y categorizadas con el software N-VIVO 10. Resultados: Emergieron 13 categorías, 182 códigos descritos en 6 ejes: el primero relacionado con el significado del cáncer y sus barreras; el segundo con las principales barreras para la atención en salud, cuyo orden de mayor a menor frecuencia fue: administrativas, económicas, culturales, de conocimiento, de comunicación e institucionales; el tercero es las estrategias para superar las barreras; el cuarto, las estrategias para disminuirlas; el quinto, sentimientos y el rol de la familia; y el sexto, necesidades del paciente. Conclusión: la fragmentación y segmentación del sistema de salud impone barreras que limitan el diagnóstico temprano del cáncer gástrico y su manejo oportuno. Además, amenazan la calidad de vida del adulto enfermo y su familia.
\end{abstract}

\section{Palabras clave}

Neoplasia gástrica, cáncer gástrico, servicios de salud, inequidades, sistema de salud.

\begin{abstract}
Introduction: Gastric cancer is a public health problem that ranks fifth in world incidence and third in mortality. Objective: The aim of the exploratory stage of this study was to describe the barriers to health care perceived by adults with gastric cancer, their caregivers, and their attending physicians in the department of Santander, Colombia in 2015 and 2016. Methodology: This is a qualitative study using process techniques and grounded theory analysis based on semi-structured interviews that were codified and categorized with N-VIVO 10. Results: Thirteen categories and 182 codes described along 6 axes emerged. The first axis is related to the meaning of cancer and its barriers. The second is related to the primary barriers to health care which are, in order from most frequent to least frequent: administrative, economic, cultural, knowledge, communication and institutional. The third axis consists of strategies to overcome barriers. The fourth consists of strategies to diminish barriers. The fifth is related to feelings and the role of the family, and the sixth contains the patient's needs. Conclusion: Fragmentation and segmentation of the health care system imposes barriers that limit early diagnosis of gastric cancer and timely management. In addition, they threaten the quality of life of the sick adult and her or his family.
\end{abstract}

Keywords

Gastric neoplasm, gastric cancer; health services, inequities, health system. 


\section{INTRODUCCIÓN}

El cáncer gástrico (CG) es una neoplasia maligna que se asocia con infección por Helicobacter pylori y con factores genéticos, ambientales, ocupacionales y dietéticos (1-7). A nivel mundial, el CG es la tercera causa de mortalidad y la quinta en incidencia con el $70 \%$ de los casos en países no desarrollados $(8,9)$. En Colombia, el CG tiene una relación hombre-mujer de 2:1 y se presenta alrededor de la séptima y octava década de vida (promedio de edad: 65 años) (10). Su incidencia y mortalidad por 100000 habitantes es de 18,9 y 15,6 en hombres; y 9,0 y 7,8 en mujeres (8). En Bucaramanga y su área metropolitana, su incidencia y mortalidad son mayores. En hombres 22,4 y 17,3 ; y en mujeres, 10,3 y 11,1 , respectivamente (11).

Los signos y síntomas del adulto con CG en la etapa inicial son inespecíficos en un $80 \%(12,13)$; estos pueden ser náuseas, vómito, regurgitación y distensión abdominal. Mientras que otros como dispepsia, dolor en epigastrio o retroesternal, pérdida de peso involuntaria, anemia y disfagia se asocian frecuentemente con CG $(14,15)$.

La oportunidad del diagnóstico depende de varios factores: el primero es el nivel de desarrollo del territorio. Los países en desarrollo tienen baja adherencia y participación en programas de tamizaje de CG comparado con Japón, donde estos programas permiten diagnosticarlo tempranamente y disminuir su mortalidad (16-19). El segundo es la condición socioeconómica del individuo. Los adultos con estrato socioeconómico bajo se automedican o no asisten a consulta oportunamente, son diagnosticados tardíamente y tienen menor supervivencia comparados con los de mayor estrato socioeconómico (20). Y el tercero es la pericia médica, que influye en el diagnóstico, tratamiento oportuno y supervivencia a 5 años; debido a que el $36 \%$ de los pacientes con CG tiene 3 o más consultas de atención primaria antes de ser diagnosticados mediante endoscopia de vías digestivas altas (EVDA) y biopsia; y el pronóstico del paciente dependerá de su estado general, edad, estadificación clínica y localización del tumor (14). En Colombia, la población subsiste con síntomas gastrointestinales más de 6 meses antes del diagnóstico, esto sugiere desconocimiento sobre la necesidad de buscar atención médica, así como baja accesibilidad a atención especializada, lo que representa barreras para la atención en el sistema general de seguridad social en salud (SGSSS) (17).

Se propuso una investigación cualitativa cuyo objetivo fue describir las barreras para la atención en salud, percibidas por adultos con CG, sus cuidadores y médicos tratantes residentes en el departamento de Santander, Colombia. Financiado por el Departamento Administrativo de Ciencia, Tecnología e Innovación (Colciencias; código 124165741298). Esto con el propósito de conocer la situación y problemática actual en los pacientes oncológicos en Colombia y específicamente en el departamento de Santander, donde es mayor la incidencia y prevalencia del CG. En este artículo se presentarán los resultados obtenidos en la etapa exploratoria de esta investigación.

\section{METODOLOGÍA}

Se abordó una metodología cualitativa basada en la interpretación y organización de los datos propuestos por la teoría fundamentada de Strauss y Corbin $(21,22)$ en una etapa exploratoria que consistió en aplicar entrevistas semiestructuradas a 9 participantes que permitieron, además de la emergencia de barreras para la atención en salud, la familiarización con la entrevista y verificación de la claridad de las preguntas, y la interacción con los datos.

\section{Participantes}

Para la selección de los participantes se realizó muestreo por conveniencia con énfasis en la calidad de los sujetos de investigación y no en la cantidad que tuvo en cuenta la percepción de quienes interactúan directamente durante la atención en salud desde la realidad que viven y el rol asumido durante la enfermedad. Se incluyeron 3 pacientes $(\mathrm{P})$ diagnosticados con CG entre enero y marzo de 2015 del Registro Poblacional de Cáncer del Área Metropolitana de Bucaramanga, Santander; 3 cuidadores (C) y 3 médicos (M) (2 gastroenterólogos y 1 cirujano oncólogo) residentes en el departamento de Santander. En total fueron 7 hombres y 2 mujeres; con promedio de edad de 53 años y desviación estándar de 10,1 años (Tabla 1).

Tabla 1. Características sociodemográficas de los participantes según su rol

\begin{tabular}{|c|c|c|c|c|c|}
\hline & Edad & Sexo & Escolaridad & Ocupación & Parentesco \\
\hline \multicolumn{6}{|c|}{ Paciente } \\
\hline 1 & 53 & Hombre & $\begin{array}{l}\text { Básica } \\
\text { primaria }\end{array}$ & Construcción & \\
\hline 2 & 64 & Hombre & $\begin{array}{c}\text { Básica } \\
\text { primaria }\end{array}$ & Agricultor & \\
\hline 3 & 57 & Hombre & Ninguna & Cesante & \\
\hline \multicolumn{6}{|c|}{ Cuidador } \\
\hline 1 & 42 & Hombre & $\begin{array}{c}\text { Básica } \\
\text { primaria }\end{array}$ & Agricultor & Cónyuge \\
\hline 2 & 45 & Mujer & $\begin{array}{c}\text { Básica } \\
\text { secundaria }\end{array}$ & Cesante & Cónyuge \\
\hline 3 & 53 & Mujer & $\begin{array}{c}\text { Básica } \\
\text { secundaria }\end{array}$ & Cesante & Hermana \\
\hline \multicolumn{6}{|c|}{ Médico } \\
\hline 1 & 40 & Hombre & Posgrado & Cirujano oncólogo & \\
\hline 2 & 52 & Hombre & Posgrado & Gastroenterólogo & \\
\hline 3 & 71 & Hombre & Posgrado & Gastroenterólogo & \\
\hline
\end{tabular}




\section{Instrumento}

Se realizaron 2 tipos de entrevista (E): una conformada por 17 preguntas para médicos y otra de 27 preguntas para adultos con CG y sus cuidadores. La estructura de los guiones fue preámbulo, ficha frontal de variables sociodemográficas y 6 ejes temáticos que responden a los objetivos específicos planteados: información sobre el cáncer, percepciones y necesidades; signos y síntomas previos al diagnóstico de cáncer; diagnóstico y tratamiento del cáncer; barreras y las respuestas dadas ante estas.

\section{Procedimiento}

Se contactó telefónicamente al médico tratante y/o la Institución Prestadora de Servicios de Salud (IPS) para contactar al adulto enfermo y su cuidador, a quienes se les explicaron los objetivos del estudio y el manejo confidencial y anónimo de la información de la entrevista. Después de su aceptación, se acordaron el lugar, la fecha y la hora para la entrevista. En la entrevista, el participante no estaba obligado a responder todas las preguntas y se le solicitó la autorización para grabar la entrevista. Ante su aprobación, se procedió a firmar el consentimiento informado y desarrollar el guión. Las entrevistas duraron en promedio 40 minutos, en un encuentro persona a persona, las cuales fueron realizadas por una psicóloga en cumplimiento de la recomendación del Comité de Ética de la Universidad Autónoma de Bucaramanga (UNAB) para mantener una conversación fluida y el manejo de crisis en caso de presentarse.

\section{Análisis}

Las entrevistas fueron transcritas y analizadas a partir de la codificación y categorización de los datos propuestas en el método de la teoría fundamentada de Strauss y Corbin con el uso del software N-VIVO 10 versión 11 para la descripción y el ordenamiento conceptual, sin tener como alcance la teorización por ser una etapa exploratoria.

Para la codificación se identificaron los atributos, cualidades y características de los datos y aquellos que compartían la misma característica fueron agrupados con un mismo nombre o código; a partir de precodificación o la subjetividad inductiva del investigador. Posteriormente, se analizó cada código, comparándolo con los demás e identificando qué tienen en común para establecer relaciones entre los códigos con el fin de agruparlos en las categorías emergentes (22).

\section{RESULTADOS}

Las categorías emergentes se describen en 6 ejes: percepciones de las palabras cáncer y barrera; barreras para la aten- ción en salud; estrategias para superar las barreras; estrategias para disminuir las barreras; y sentimientos, el rol de la familia y necesidades del paciente.

En el primer eje para los participantes, el significado de cáncer se relacionó con muerte, minusvalía e intimidación, malignidad y designio de Dios; y el de barrera con obstáculo. El segundo eje está conformado por 6 barreras para la atención en salud. Las barreras administrativas se orientaron a la percepción de la respuesta obtenida durante la atención en salud de signos y síntomas, como tratamientos sintomáticos por tiempo prolongado sin seguimiento $y$ ausencia de exámenes y procedimientos. Se comentó en las entrevistas del siguiente modo:

"Usualmente formulan para la gastritis y no hacen la endoscopia, no piden ningún examen. Pienso que el principal factor está ahí en que el paciente se trata con base en los síntomas y signos generales sin estudio” EM.

Durante el diagnóstico de la enfermedad, las barreras administrativas se identifican en situaciones como la demora para autorizar exámenes y procedimientos, específicamente la EVDA y los trámites para recibir atención especializada. Esto genera en el paciente sentimientos de insatisfacción y abandono. Para los médicos, los problemas administrativos son obstáculos impuestos por el SGSSS frente a su criterio emitido en las órdenes médicas por priorizar el gasto en salud sobre la calidad de vida, como se manifestó en los testimonios:

"Lo mantienen a uno abandonado (...). La EPS me demoró mucho y me pude morir. Lo único que saben es cobrar, me pusieron a hacer muchas vueltas, entonces yo no me siento bien por eso." EP.

EPS: empresa promotora de servicios de salud.

Durante el tratamiento, las barreras administrativas son el resultado de la fragmentación institucional del SGSSS que conllevan a trámites para autorizar citas médicas o procedimientos que, en algunos casos, son negados por la aseguradora y ocasionan una derivación a particulares. Esto revela la ausencia de vigilancia del Estado en los contratos e instituciones que atienden adultos con CG. Se comentó en las entrevistas del siguiente modo:

"La gente se aburre de hacer fila. Además, la cita se la dan para dentro de 2 meses y se devuelven al pueblo sintiendo que perdieron el viaje." EM.

Las barreras económicas se orientan a la capacidad que tienen las personas de cubrir las necesidades secundarias a la 
enfermedad. Para los entrevistados, la pobreza limita el uso del dinero como satisfactor e identifican el gasto de bolsillo como empobrecedor, debido al pago de atención en salud y transporte en una condición de difícil ahorro; y en algunos casos, el ausentismo laboral presiona a los pacientes a suspender el tratamiento.

Las barreras culturales se evidencian en las respuestas brindadas ante la necesidad de atención en salud como la búsqueda de medicina alternativa y/o naturista, la asistencia a droguerías y a médicos por sugerencia. Se narró en las entrevistas de la siguiente manera:

"El hombre campesino es quien toma las determinaciones así sean equívocas, como acudir a medicina alternativa, donde dicen que lo curan todo." EM.

Las barreras de conocimiento identificadas son la dificultad que tiene el médico, de nivel primario, para asociar los signos y síntomas inespecíficos o su persistencia con CG referidos por el paciente y cuidador como vómito, ardor, picadas, pérdida de peso y gastritis. También, se identifica que la atención primaria se resume en tratamientos sintomáticos y, en algunos casos, remisión al especialista (gastroenterólogo). Según se narra en los siguientes testimonios:

"Un médico no necesita ser un científico sino una persona con concepto integral en salud que debe conocer los fundamentos semiológicos de la enfermedad, tener juicio, raciocinio y conocer la epidemiología del cáncer gástrico." EM.

Las barreras de comunicación son fallas en la relación médico-paciente atribuidas a la falta de credibilidad y confianza en el lenguaje, información y explicación que proporciona el profesional de la salud al paciente $y$ a su familia e influye en su compresión y aceptación de la enfermedad. Como se narra en seguida:

"El $100 \%$ de mis pacientes con cáncer lo saben, yo les digo a todos todo. Dependiendo del nivel cultural uso la forma más cómoda para explicarles que tienen una enfermedad grave" EM.

Las barreras institucionales responden al tiempo excesivo para la adjudicación de citas médicas que requieren estrategias como súplica e insistencia, que no son priorizadas por la complejidad del cuadro clínico. Los participantes perciben que la duración del proceso de atención en salud entre síntomas y diagnóstico puede ser de 12 a 18 meses, y el tiempo entre el diagnóstico y el inicio de tratamiento varía entre 1 a 6 meses.
Adicionalmente, las alternativas para superar las barreras identificadas por los médicos son la educación, la atención integral (juntas médicas interinstitucionales) y el ingreso del paciente a urgencias. Para los pacientes, sus estrategias son las creencias religiosas y la fe en Dios. En cambio, para los cuidadores es conseguir dinero para satisfacer las necesidades apremiantes.

Para disminuir las barreras de acceso a la atención en salud se requiere crear centros médicos especializados en oncología soportados por el Estado y dirigir los recursos para garantizar y rectificar el proceso de atención en salud suprimiendo la tramitología.

Por una parte, según la percepción del paciente sobre su condición, el adulto enfermo señala como una de las consecuencias más fuertes el retiro laboral, la disminución de su capacidad de generar ingresos y la insatisfacción de necesidades. Asimismo, siente desprotección de las EPS, pero resalta la importancia del apoyo moral de la familia, el soporte económico y su importancia como gestores de la atención en salud. Por otra parte, el médico y los cuidadores perciben que las necesidades son la educación para pacientes y cuidadores dirigida hacia la motivación y el direccionamiento de la atención en salud. Y para el paciente y el cuidador son la atención médica oportuna e integral, soporte económico, ayuda de la aseguradora para costear el albergue y el cuidado domiciliario.

\section{DISCUSIÓN}

Según este estudio, las barreras para la atención en salud percibida por los participantes (adultos con CG, sus cuidadores y médicos tratantes) obedecen a la condición del individuo o son secundarias a la estructura del SGSSS. La identificación de estas barreras permite correlacionarlas con el impacto en la mortalidad y la calidad de vida de la población del Área Metropolitana de Bucaramanga, e influyen en el pronóstico del CG porque agudizan los sentimientos de estrés y ansiedad, reducen la calidad de vida del adulto enfermo y, potencialmente, afectan la supervivencia (23).

Las barreras para la atención médica en países diferentes a Colombia se clasifican en 4 componentes: aceptabilidad, accesibilidad, contacto y disponibilidad; estas son similares a los hallazgos del presente estudio. Por ejemplo, la aceptabilidad según Adauy y colaboradores es el temor o vergüenza de asistir al servicio de salud, desconfianza en los profesionales de salud y tratamiento prescrito (24); creencias y mitos que influyen en la percepción y respuesta ante el cáncer. En Colombia, por ejemplo, la búsqueda de atención médica depende del régimen de afiliación (especial: 15,7\%, contributivo: $21,1 \%$, subsidiado: $30,6 \%$ y no asegurado: $57,75 \%$ ) $\mathrm{y}$, en respuesta, los pacientes se automedican orientados por 
familiares y farmaceutas con una mejoría transitoria y retraso en el diagnóstico (25-28). El 89,7 \% de 428 adultos con cáncer declaró haber adquirido medicamentos sin prescripción médica por falta de tiempo para consultar, demora en la atención y dificultades de acceso a la institución en relación con su residencia (29). Ante estas barreras, la respuesta de pacientes y cuidadores es usar medicina no tradicional y remedios caseros $(26,30-33)$.

Lo anterior está asociado con las barreras en accesibilidad ocasionada por los tiempos de espera excesivos (24) debido a que la atención en salud en Colombia es fragmentada, mediada por trámites para autorizar citas médicas y procedimientos que, en ocasiones, se niegan por ausencia de contratación entre las aseguradoras y los diferentes proveedores de servicios de salud $(30,34)$. Esto limita el seguimiento de la enfermedad, como se evidenció en las entrevistas, que genera en los pacientes y cuidadores la percepción de baja capacidad de respuesta del SGSSS y aumento del gasto de bolsillo $(25,29,35)$.

\section{CONCLUSIÓN}

La identificación de las barreras para la atención en salud en CG genera estrategias dirigidas a superarlas; más aún, cuando las barreras más frecuentes fueron de origen administrativo secundarias a la segmentación institucional y fragmentación operativa del SGSSS. Para ello se requiere la orientación de los servicios de salud hacia una atención integral que sobrepase la lógica de mercado del sistema y favorezca la operatividad del SGSSS con epicentro en el individuo, su entorno y sus determinantes sociales. Esto impacta en la reducción de los tiempos de atención en salud para aumentar la supervivencia de los adultos con CG.

\section{LIMITACIONES}

El estudio no permite cuantificar el impacto de las barreras para la atención en salud del CG en la mortalidad y en la supervivencia debido al método de estudio. Se sugieren estudios posteriores que correlacionen las barreras para la atención en salud del CG con su supervivencia en Bucaramanga y su área metropolitana.

\section{Agradecimientos}

Agradecemos a Colciencias, que apoyó la realización del estudio.

\section{Consideraciones éticas y conflicto de intereses}

$\mathrm{Al}$ estudio realizado no le atañen consideraciones éticas relativas a los casos clínicos y a la intervención de pobla- ción; sin embargo, es preciso mencionar que las autoras no incurren en faltas éticas respecto a las fuentes referenciadas, pues se atienden a las pautas pertinentes de citación y protección de la propiedad intelectual. Asimismo, las autoras declaran no tener conflicto de intereses que afecten esta publicación.

\section{Fuente de apoyo financiero}

Colciencias.

\section{REFERENCIAS}

1. Torre LA, Siegel RL, Ward EM, Jemal A. Global Cancer Incidence and Mortality Rates and Trends--An Update. Cancer Epidemiol Biomarkers Prev. 2016;25(1):16-27. doi: 10.1158/1055-9965.EPI-15-0578.

2. Goral V. Etiopathogenesis of Gastric Cancer. Asian Pac J Cancer Prev. 2016;17(6):2745-50.

3. McColl KE. Clinical practice. Helicobacter pylori infection. N Engl J Med. 2010;362(17):1597-604. doi: 10.1056/ NEJMcp1001110.

4. Raei N, Behrouz B, Zahri S, Latifi-Navid S. Helicobacter pylori Infection and Dietary Factors Act Synergistically to Promote Gastric Cancer. Asian Pac J Cancer Prev. 2016;17(3):917-21.

5. Massarrat S, Stolte M. Development of gastric cancer and its prevention. Arch Iran Med. 2014;17(7):514-20. doi: 0141707/AIM.0013.

6. Jayalekshmi PA, Hassani S, Nandakumar A, Koriyama C, Sebastian P, Akiba S. Gastric cancer risk in relation to tobacco use and alcohol drinking in Kerala, India--Karunagappally cohort study. World J Gastroenterol. 2015;21(44):1267685. doi: 10.3748/wjg.v21.i44.12676.

7. Wen XY. Salt taste sensitivity, physical activity and gastric cancer. Asian Pac J Cancer Prev. 2010;11(6):1473-7.

8. World Health Organization. International Agency for Research on Cancer. GLOBOCAN; 2012: Estimated Cancer Incidence, Mortality and Prevalence Worldwide in 2012. Population Fact Sheets. GLOBOCAN [internet] 2012 [acceso el 10 de octubre de 2016]. Disponible en: http://globocan.iarc.fr/Pages/fact_sheets_population.aspx.

9. Chang Y, Cho B, Son KY, Shin DW, Shin H, Yang HK, et al. Determinants of gastric cancer screening attendance in Korea: a multi-level analysis. BMC Cancer. 2015;15:336. doi: 10.1186/s12885-015-1328-4.

10. Blanco O, Cantillo A, Rivera J. Enfoque actual del cáncer gástrico. Medicas UIS. 2013;26(2):59-70.

11. Uribe C, Osma S, Herrera V. Cancer incidence and mortality in the Bucaramanga metropolitan area, 2003-2007. Colomb Médica. 2012;43(4):290-7.

12. García C. Actualización del diagnóstico y tratamiento del cáncer gástrico. Rev Méd Clin Condes. 2013;24(4):627-36.

13. Gómez MA, Riveros JH, Ruiz O, Concha A, Betamcur DMA, Torres M, et al. Guía de práctica clínica para la pre- 
vención, diagnóstico y tratamiento del cáncer gástrico temprano - 2015. Rev Colomb Cancerol. 2015;30(1):34-42.

14. Astin MP, Martins T, Welton N, Neal RD, Rose PW, Hamilton W. Diagnostic value of symptoms of oesophagogastric cancers in primary care: a systematic review and meta-analysis. Br J Gen Pract. 2015;65(639):e677-91. doi: 10.3399/bjgp15X686941.

15. Mayer RJ. Upper gastrointestinal tract cancers. En: Kasper D, Fauci A, Hauser S, Longo D, Jameson J, Loscalzo J (editores). Harrison's Principles of Internal Medicine. 19. ${ }^{a}$ edición. Nueva York: McGraw-Hill; 2014. p. 447-60.

16. Calvo A. Diagnóstico precoz del cáncer gástrico: estrategias de prevención secundaria y dificultades del diagnóstico de lesiones precoces. Rev Méd Clin Condes. 2011;22(4):477-84.

17. Oliveros R, Navarrera LF. Diagnóstico, estadificación y tratamiento del cáncer gástrico en Colombia desde 2004 a 2008 (REGATE-Colombia). Rev Colomb Gastroenterol. 2012;27(4):269-74.

18. Lee KJ, Inoue M, Otani T, Iwasaki M, Sasazuki S, Tsugane S; et al. Gastric cancer screening and subsequent risk of gastric cancer: a large-scale population-based cohort study, with a 13-year follow-up in Japan. Int J Cancer. 2006; 118(9):231521. doi: $10.1002 / \mathrm{ijc} .21664$.

19. Higashi T, Machii R, Aoki A, Hamashima C, Saito H. Evaluation and revision of checklists for screening facilities and municipal governmental programs for gastric cancer and colorectal cancer screening in Japan. Jpn J Clin Oncol. 2010;40(11):1021-30. doi: 10.1093/jjco/hyq091.

20. Siemerink EJ, Hospers GA, Mulder NH, Siesling S, van der Aa MA. Disparities in survival of stomach cancer among different socioeconomic groups in North-East Netherlands. Cancer Epidemiol. 2011;35(5):413-6. doi: 10.1016/j. canep.2011.02.015.

21. Noerager P, Jane C. Essential of accesible grounded theory. 1. a edición. Estados Unidos: Left Coast Press; 2011. p. 42.

22. Strauss A, Corbin J. Bases de la investigación cualitativa, técnicas y procedimientos para desarrollar la teoría fundamentada. 1. ${ }^{a}$ edición. Colombia: Editorial Universidad de Antioquia; 2002. p. 11-28.

23. Hamlyn GS, Hutchins KE, Johnston AL, Thomas RT, Tian J, Kamal AH. Accessibility and Barriers to Oncology Appointments at 40 National Cancer Institute-Designated Comprehensive Cancer Centers: Results of a Mystery Shopper Project. J Oncol Pract. 2016;12(10):e884-e900. doi: 10.1200/JOP.2016.014159.
24. Adauy M, Poffald L, Jasmen AM, Aguilera X, Delgado I, Vega J. Barreras y facilitadores de acceso a la atención de la salud: una revisión sistemática cualitativa. Rev Panam Salud Pública. 2013;33(3):223-9.

25. Garcia-Subirats I, Vargas I, Mogollón-Pérez AS, De Paepe P, da Silva MR, Unger JP, et al. Barriers in access to healthcare in countries with different health systems. A cross-sectional study in municipalities of central Colombia and north-eastern Brazil. Soc Sci Med. 2014;106:204-13. doi: 10.1016/j. socscimed.2014.01.054.

26. Kagawa-Singer M, Dadia AV, Yu MC, Surbone A. Cancer, culture, and health disparities: time to chart a new course? CA Cancer J Clin. 2010;60(1):12-39. doi: 10.3322/caac.20051.

27. Pinzón CE, Ospina J. Dispepsia: un real desafío diagnóstico. Rev Cienc Salud Bogotá. 2008;6(1):50-70.

28. Galindo F. Carcinoma gástrico. Cirugía digestiva. 2009;II223:1-31.

29. Del Toro M, Díaz A, Barrios Z, Castillo IY. Automedicación y creencias en torno a su práctica en Cartagena, Colombia. Rev Cuid. 2017;8(1):1509-18.

30. Restrepo JH, Silva C, Andrade F, Vh R. Acceso a servicios de salud: Análisis de barreras y estrategias en el caso de Medellín, Colombia. Rev Gerenc y Polit Salud. 2014;13(27):242-65. doi: 10.11144/Javeriana.rgyps13-27.assa.

31. Hernández A, Vega R. El sistema colombiano de seguridad social en salud: desigualdad y exclusión. Rev Gerenc y Políticas Salud. 2001;1(1):48-73.

32. Hunt R, Quigley E, Abbas Z, Eliakim A, Emmanuel A, Goh KL, et al. Manejo de los síntomas gastrointestinales frecuentes en la comunidad. Perspectiva mundial sobre acidez, estreñimiento, distensión y dolor o molestia abdominal. Guías Mundiales de la Organización Mundial de Gastroenterología; 2013.

33. Woods LM, Rachet B, Coleman MP. Origins of socio-economic inequalities in cancer survival: a review. Ann Oncol. 2006;17(1):5-19. doi: 10.1093/annonc/mdj007.

34. Garcés IC, Rubio DC, Ramos SM. Barreras y facilitadores del sistema de salud relacionadas con el seguimiento de anormalidades citológicas, Medellín-Colombia. Rev Gerenc y Polit Salud. 2014;13(27):200-11. doi: 10.11144/ Javeriana.rgyps 13-27.bfss.

35. Vargas I, Vázquez ML, Mogollón AS. Acceso a la atención en salud en Colombia. Rev Salud Pública. 2010;12(5):701-12. doi: 10.1590/S0124-00642010000500001. 